\title{
Craniofacial surgery for craniometaphyseal dysplasia
}

\author{
F. U. Ahmad, A. K. Mahapatra, H. Mahajan \\ Department of Neurosurgery, Neurosciences Centre, All India Institute of Medical sciences, New Delhi, India
}

\begin{abstract}
Craniometaphyseal dysplasia $(\mathrm{CMD})$ is a rare congenital bone dysplasia with abnormal bony overgrowth leading to characteristic facial features and cranial nerve compression. We present a 10-year-old child with bony swelling at the nasal root since birth along with decreased hearing in both ears. She had normal developmental milestones and intelligence. On examination, she had bossing of forehead with very broad nasal root, short septum, hypertelorism and epicanthic folds. CT scan with 3D reconstruction revealed grossly thickened calvarium and hyperostosis and sclerosis of the cranial base. As the major concern of the parents was cosmetic, craniofacial reconstruction was performed with good cosmetic outcome
\end{abstract}

Key words: Craniofacial remodeling, craniometaphyseal dysplasia.

Craniometaphyseal dysplasia (CMD) is a rare congenital bone dysplasia characterized by sclerosis of the skull bones, hyperostosis of skull base, frontal, facial and occipital bones and widening of the metaphyseal region of long bones. ${ }^{[1]}$ Abnormal bony overgrowth leads to characteristic facial features and cranial nerve compression. We present a case that was referred to the Department of Neurosurgery as a case of fronto-nasal encepahalocele but turned out to be CMD, though the metaphyseal changes were not overt at this age. Amongst 300 cases of craniofacial surgery performed at our Department in the last 30 years,${ }^{[2]}$ this is the first case of $\mathrm{CMD}$, which stimulated us for this publication.

\section{Case Report}

A 10-year-old child presented to our department with bony swelling at the nasal root since birth. She also complained of decreased hearing in both ears and failing vision in both eyes since childhood, both of which had stabilized over the last 2 years. She had normal developmental milestones and there was no history of seizures, nasal obstruction, paranasal sinusitis or epiphora. Family history was noncontributory with nonconsanguineous parents. On examination, she had bossing of forehead with bi-temporal narrowing, very broad nasal root with short septum, grade 3 hypertelorism with intercanthal distance of $45 \mathrm{~mm}$ and epicanthic folds [Figure 1]. She had a flat philtrum with high arched palate but no cleft. Her external occipital protuberance was unusually prominent. Her vision was 6/36 in both eyes with left facial paresis and bilateral sensorineural hearing loss. Eye movements and visual evoked responses were normal. Rest of the neurological examination was essentially normal. There were no other bony or skeletal abnormalities and no organomegaly. Pure tone audiometry revealed bilateral moderate sensorineural hearing loss. Brainstem auditory evoked responses showed delayed wave 1 with normal

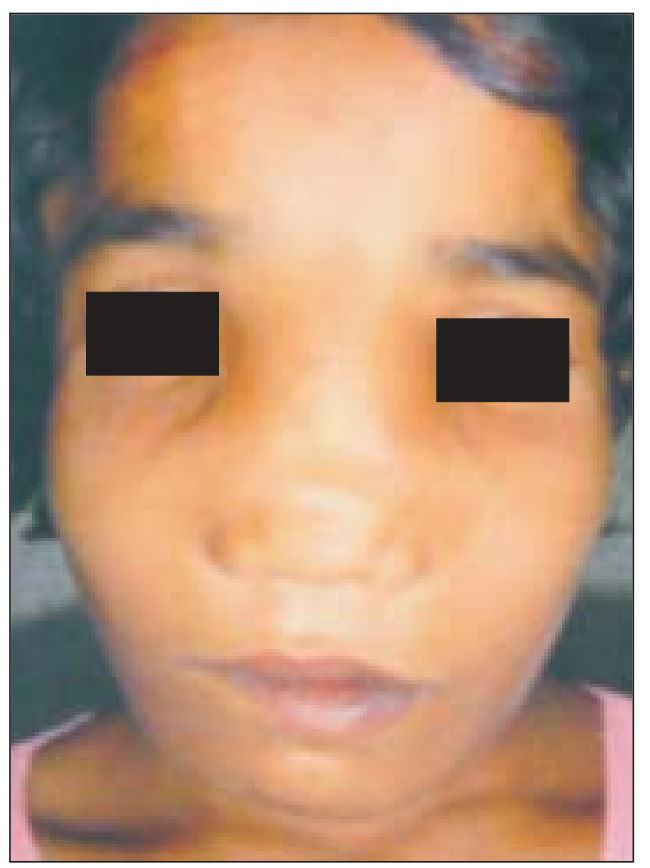

Figure 1: Child with craniometaphyseal dysplasia showing widening of nasal bridge and prominent cheek bones

\section{A. K. Mahapatra}

7th floor, Department of Neurosurgery, Neurosciences Centre, All India Institute of Medical sciences, New Delhi - 110029 , India.

E-mail: akmahapatra_22000@yahoo.com 
amplitude and interpeak latencies.

$\mathrm{CT}$ scan with axial, coronal and $3 \mathrm{D}$ reconstruction [Figure $2 \mathrm{~A}-$ C] revealed diffusely thickened calvarium and hyperostosis and sclerosis of the cranial base and maxilla. The nasal bridge, zygoma, frontal bones and inion were unduly prominent, with narrowing of the optic foramina and superior orbital fissures. Cerebral hemispheres, posterior fossa structures and parapharyngeal spaces are normal.

As the major concern of the parents was cosmetic, the craniofacial remodeling was planned after explaining in detail to the parents. With the patient in supine position under general anesthesia, a bicoronal skin flap was raised. Remodeling of forehead was carried out first to make the forehead cosmetically acceptable. The frontal bone was 1-1.5-cm thick. The nasal bone, ethmoid and frontal process of maxilla were very bulky and unduly prominent. The thick and sclerotic bones were recontoured using Midas-Rex drill (AM-8 attatchment). Dysplastic bone was found to be extremely thick, ivory hard and mineralized and its removal required lengthy and extensive drilling. Drilling was also carried out at the medial canthus so that the intercanthal distance was reduced. The inner canthus was reconstructed by stitching the intercanthal ligament to the fronto-nasal junction on the either side.

Patient had a $4 \mathrm{~h}$ surgery with $800 \mathrm{ml}$ of blood loss, which was adequately replaced. She had an uneventful postoperative period and was discharged from the hospital on seventh postoperative day. CT scan of head with surface reconstruction, done 9 months later, showed reduction in size of the frontal and nasal bones [Figure 3].

\section{Discussion}

The term CMD was coined by Jackson et $\mathrm{al}^{[3]}$ in 1954 , for a hereditary bone disease with metaphyseal widening of the tubular bones and bony overgrowth of the facial and skull bones (leonteasis ossea). It is characterized by disordered intramembranous and endochondral ossification. ${ }^{[4]}$ Ear and nasal symptoms are the presenting complaints in two-thirds of the cases. ${ }^{[5]}$ Craniofacial abnormalities are prominent and include frontonasal bossing, hypertelorism, prognathic mandible and defective dentition. Intelligence is usually normal. Bony compression can involve various cranial nerves, which can result in blindness, strabismus, facial nerve paresis and deafness. Quadriparesis or death may result from foramen magnum narrowing and compression of medulla in the severe forms of the disease. ${ }^{[4,6]}$

The major differential diagnosis of CMD are metaphyseal dysplasia (Pyle's disease) and craniodiaphyseal dysplasia (CDD) ${ }^{[1]}$ In the former, the skull changes are less overt with prominent metaphyseal widening and pelvic bones are also involved. In the latter, there is more significant flaring of the diaphyseal region and severe sclerosis and hyperostosis of the facial and skull bones without much of metaphyseal involvement. Both of these are inherited as autosomal recessive whereas CMD can be either autosomal recessive or dominant. ${ }^{[1,7]}$ The differentiation is important as the natural progression and prognosis is significantly worse in CDD.

The management of such cases, especially those at the severe end of spectrum with massive craniofacial hyperostosis such as present in our case, is extremely difficult.

Medical management in an attempt to influence the hyperostosis has been attempted with calcitriol, low calcium intake, bisphosphonates and also somatostatin. None of these have resulted in convincingly beneficial results. ${ }^{[6,8]}$ The low calcium diet is likely to prevent further deposition of mineral bone and allow the calcitriol induced osteoclasts to reduce the skull bone mass. ${ }^{[9]}$ Somatostatin may have a role in slowing the progressive hyperostosis. ${ }^{[6]}$

Surgical results in this condition are inconsistent, as reported in literature. ${ }^{[4,6,8,10-12]}$ Reports are available stating rapid bone growth following surgery. ${ }^{[6]}$ Optic nerve decompression has been reported in literature but the results are not encouraging, with reduction of vision and blindness reported after optic canal
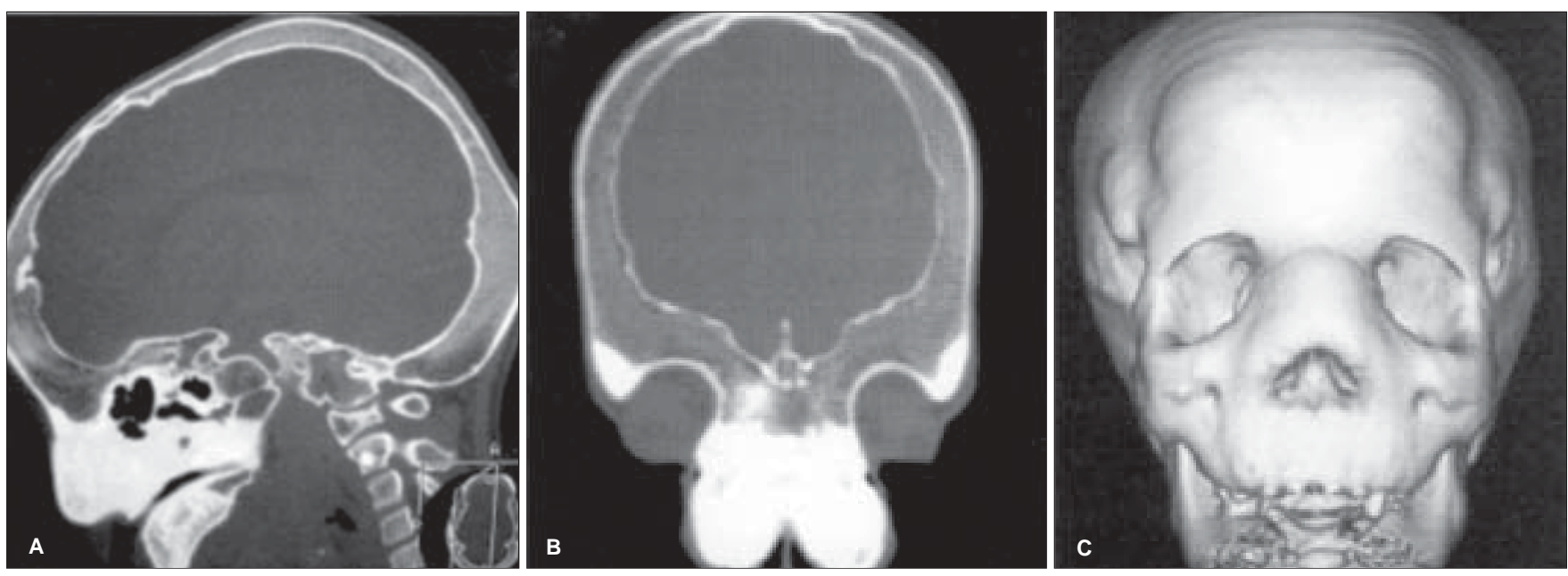

Figure 2: (A-C): CT scan with saggital, coronal and 3D reconstruction revealed diffusely thickened skull vault with hyperostosis and sclerosis of the cranial base and maxilla. The nasal bridge, zygoma and frontal bones are unduly prominent 


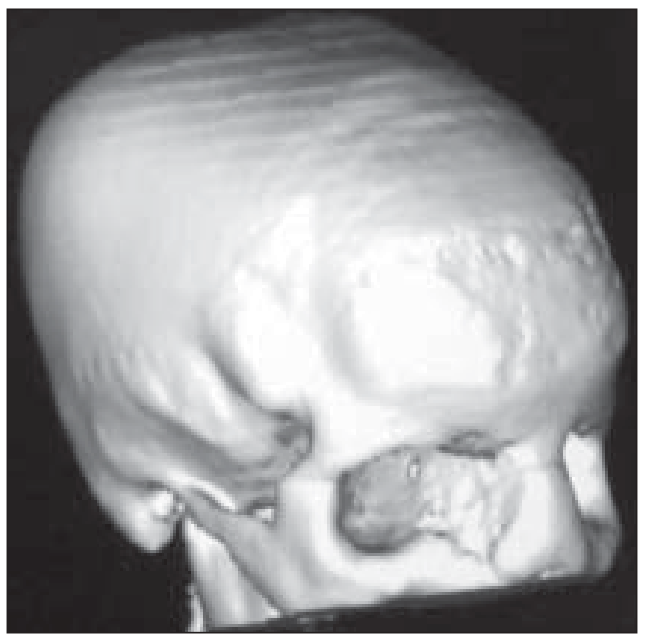

Figure 3: Postoperative CT scan with 3D reconstruction showing reduction in size of the thick bones

decompression. ${ }^{[10,11,13]}$

Facial nerve involvement results from bony compression inside the petrous temporal bone. Facial nerve decompression has never been reported. Richards et al. do not recommend it as the benefits are likely to be short-lived and the technical difficulties and risks are enormous (sclerotic mastoid, ivory hard bone and obscured bony landmarks due to bony overgrowth). ${ }^{[6]}$ The same difficulties are likely to be encountered during decompression of internal auditory meatus for the treatment of sensorineural hearing loss. Day et al. reported good surgical result in a patient presenting with CMD, foramen magnum stenosis, Chiari I malformation and syringomyelia. ${ }^{[4]}$ Foramen magnum decompression was performed via suboccipital craniectomy, C1-C2 laminectomy and dural augmentation. These patients are usually treated by ophthalmologists and otolaryngologists.

The neurosurgeons and craniofacial surgeons are usually consulted in such cases for craniofacial deformities and neurological symptoms. It is important for them to realize that the surgical intervention in such cases is palliative, technically difficult due to ivory hard bone and the benefits may be shortlived. Cranial nerve decompression in such cases, though described in literature, is fraught with danger and the risks should be explained to the caretakers. However, cervical spinal cord/medullary compression by bony overgrowth should be undertaken as it portends an impending threat to life. The pathophysiology is of the disease is still incompletely understood. Early diagnosis is important to allow early commencement of medical therapy as well as explaining the prognosis of the disease and its treatment to the parents and relatives.

\section{References}

1. Cheung VG, Boechat MI, Barrett CT. Bilateral choanal narrowing as a presentation of craniometaphyseal dysplasia. J Perinatol 1997;17:241-3.

2. Mahapatra AK, Suri A. Anterior encephaloceles: a study of 92 cases. Pediatr Neurosurg 2002;36:113-8

3. Jackson WPU, Albright F, Dewry G, Hanelin J, Rubin MI. Metaphyseal dysplasia, epiphyseal dysplasia, diaphyseal dysplasia and related conditions. Arch Int Med 1954;94:871-85.

4. Day RA, Park TS, Ojemann JG, Kaufman BA. Foramen magnum decompression for cervicomedullary encroachment in craniometaphyseal dysplasia: case report. Neurosurgery 1997;41:960-4.

5. Martin FW. Otorhinolaryngological aspects of craniometaphyseal dysplasia. Clin Otolaryngol 1979;4:67-76.

6. Richards A, Brain C, Dillon MJ, Bailey CM. Craniometaphyseal and craniodiaphyseal dysplasia, head and neck manifestations and management. J Laryngol Otol 1996;110:328-38.

7. Kirkpatrick DB, Rimoin DL, Kaitila I, Goodman S.J. The craniotubular bone modeling disorders: A neurosurgical introduction to rare skeletal dysplasia with cranial nerve compression. Surg Neurol 1977;7:221-32.

8. Elcioglu N, Hall CM. Temporal aspects of craniometaphyseal dysplasia: Autosomal recessive type. Am J Med Genet 1998;76:245-51.

9. Key LL Jr, Volberg F, Baron R, Anast CS. Treatment of craniometaphyseal dysplasia with high dose calcitriol. J Pediatr 1988;112:583-6.

10. Puliafito CA, Wray SH, Murray JE, Boger WP $3^{\text {rd }}$. Optic atrophy and visual loss in craniometaphyseal dysplasia. Am J Opthal 1981;92:696-701.

11. Satoh K, Iwata T, Ikeda H. Unsucessful consequence of optic canal decompression for a case of craniometaphyseal dysplasia. Plast Reconstr Surg 1994;94:705-8.

12. Sheppard WM, Shprintzen RJ, Tatum SA, Woods CI. Craniometaphyseal dysplasia: a case report and review of medical and surgical management. Int J Pediatr Otorhinolaryngol 2003;67:687-93.

13. Puri P, Chan J. Craniometaphyseal dysplasia: ophthalmic features and management. .J Pediatr Ophthalmol Strabismus 2003;40:228-31.

Accepted on 23-07-2005 